\title{
Relación conductual de la motivación y el autoconcepto físico en el consumo de drogas de adolescentes deportistas \\ Behavioral relationship between motivation and physical self-concept in drug use among adolescent athletes \\ Pablo Usán Supervía, Carlos Salavera Bordás, Víctor Murillo Lorente,Abel Merino Orozco \\ Universidad de Zaragoza (España)
}

Resumen: El consumo de drogas en nuestro país es uno de los problemas más graves a los que se enfrenta la sociedad actual, especialmente relevante en población adolescente (Becoña, 2007; Calafat et al., 2011; Hock y Weil, 2013 y PNSD, 2013). La práctica de la actividad físico-deportiva es considerada uno de los hábitos más representativos de un estilo de vida saludable (Cruz, Fernández y González, 2007) pudiendo actuar como una factor preventivo del consumo (De Vries et al., 2006; Warren et al., 2008). La motivación y autoconcepto físico de los deportistas jugarán un papel fundamental en la adherencia a la misma (García-Calvo, 2006; Macarro, Romero y Torres, 2008). De esta manera, el estudio tiene como objetivo analizar la relación e influencia de la motivación y autoconcepto físico en el consumo de alcohol, tabaco y cannabis en una muestra de 512 adolescentes deportistas españoles (M=16,01; DT=1,465). Los resultados desprenden influencias significativas de motivaciones intrínsecas, amotivación, motivación extrínseca identificada y competencia percibida en los diferentes consumos. Implicaciones prácticas a la hora de promover comportamientos autodeterminados en adolescentes deportistas.

Palabras clave: Motivación, autoconcepto físico, consumo de drogas, adolescentes.

Abstract: The use of drugs in our country is one of the most severe problems that our society is currently facing, especially among the adolescent population (Becoña, 2007; Calafat et al., 2011; Hock y Weil, 2013 y PNSD, 2013). Involvement in physical and sports activities is considered to be one of the most representative forms of a healthy lifestyle (Cruz, Fernández y González, 2007), as they act as a preventative factor of drug use (De Vries et al., 2006; Warren et al., 2008). Athletes' motivation and physical self-concept play a fundamental role for their adherence to these activities (García-Calvo, 2006; Macarro, Romero y Torres, 2008). Consequently, the aim of this research is to analyse the relationship and influence of motivation and physical self-concept on alcohol, tobacco and cannabis consumption in a sample of 512 Spanish adolescent athletes (M=16,01; $\mathrm{DT}=1,465)$. Results establish significant influences of intrinsic motivation, amotivation, extrinsic motivation, and perceived competence, on all the type of consumptions evaluated. Our outcomes have practical implications for the promotion of self-determined behaviour among adolescent athletes.

Keywords: Motivation, physical self-concept, drug use, adolescent.

\section{Introducción}

El consumo de drogas en nuestro país ha experimentado un aumento significativo desde principios de la década de los noventa hasta nuestros días ligado a un cambio en los valores de nuestra sociedad, de la misma manera que a un considerable incremento de consecuencias sociales, sanitarias y culturales (Baquero, Pastor y Llopis, 2003; Calafat, Juan, Becoña, Fernández y Gil, 2002; Oliva, Parra y Sánchez-Queija, 2006).

En nuestros días, conocemos que el consumo de drogas, tanto las de tipo legal (alcohol, tabaco, tranquilizantes, etc.), como las de tipo ilegal (marihuana, hachís, cocaína, anfetaminas, éxtasis, alucinógenos, etc.) poseen una desigual prevalencia de consumo pudiendo derivar en problemas para la salud de las personas y una creciente mortalidad en muchos de sus consumidores jóvenes y adultos (Becoña, 2007). Según el Observatorio Español de la Droga y las Toxicomanías (OEDT, 2016) las drogas más consumidas por los españoles adolescentes quedan encabezadas por el alcohol, seguidas del tabaco y cannabis, respectivamente.

Esta problemática ha supuesto una preocupación social y ha conducido a una profunda transformación y adecuación de las estructuras, recursos y programas dirigidos desde la Administración con el objetivo de poder hacer frente a las necesidades planteadas desde diferentes ámbitos como son la prevención, especialmente en edades tempranas, el control de la oferta de sustancias adictivas y/o la asistencia sanitaria y social (PNSD, 2013). Los costes que ello acarrea, tanto a nivel económico como personal, familiar y social son enormes, atentando de una manera directa contra la persona y su entorno más próximo (Boyle y O’Sullivan, 2013; OEDT, 2016).

De este modo, las drogas constituyen uno de los problemas más importantes con los que se enfrenta la sociedad actual, siendo especialmente preocupante cuando el consumo se produce en población infantil

Fecha recepción: 11-06-16. Fecha de aceptación: 16-03-17 Pablo Usán Supervía

pablousan@hotmail.com y juvenil, no sólo por los problemas inmediatos que puede generar sino también por la consolidación del consumo que conlleva su inicio desde edades tempranas (Becoña, 2007; Capdevila, Bellmunt y Hernando, 2015; Hock y Weil, 2012).

Desde el Plan Nacional Sobre Drogas (PNSD, 2013), se confirma que en los últimos años no sólo han variado los porcentajes globales de consumo en un aumento significativo entre adolescentes sino que también se han producido variaciones en la edad de inicio al mismo. Alcohol, tabaco y cannabis, por ese orden, resultan las sustancias más consumidas por los adolescentes españoles. Otro aspecto a resaltar es el hecho de que los consumidores actuales no sólo consumen un tipo de sustancia, sino que incluyan otras en sus pautas de consumo. Por ejemplo, es frecuente que los consumidores de cocaína consuman, además de tabaco y alcohol, otras drogas ilegales como cannabis o alucinógenos (Calafat el al., 2002). De esta manera, nos encontramos ante una situación compleja en la que un gran porcentaje de la población consumidora abusa no sólo de una sustancia sino de varias al mismo tiempo.

Distintos estudios señalan que existe un porcentaje relativamente alto de escolares de Educación Secundaria que ha tenido contacto con distintas drogas, sobre todo con alcohol, tabaco y cannabis (Rodríguez, De Abajo y Márquez, 2004; Espada, Hidalgo y Méndez, 2000; Goncy y Mrug, 2013; Hock y Weil, 2012) y que éstas pueden servir de acceso al consumo de otras sustancias (Usán, 2014), conduciendo unas a las otras (Boyle y O'Sullivan, 2012; Dunn y Thomas, 2013; Salamó, Gras y Font-Mayolas, 2010).

Trabajos recientes insisten en que se trata de un problema complejo en el que interaccionan variables de muy diversa índole (Bravo, Echeburúa y Azpiri, 2008; Espada, Méndez, Griffin y Botvin, 2003; Gilman et al., 2009; Ingles et al., 2007).

Sin duda, resulta una situación realmente preocupante si tenemos en cuenta el peso de la adolescencia en la vida de una persona, considerándola un periodo vital en el cual tienen lugar los primeros contactos con las sustancias adictivas que pueden derivar en la instauración y consolidación de patrones estables de consumo e, incluso, la aparición temprana de problemas relacionados con la salud, autoconcepto, 
autoestima, y ajuste social, que pueden llegar a afectar de manera determinante en la edad adulta (Alcalá, Azañas, Moreno y Gálvez, 2002; Gómez-Fraguela, Fernández, Romero y Luengo, 2008; Kyle, Mendo, Garrido y Sánchez, 2016).

Por otro lado, la práctica de la actividad física y deportiva está considerada como uno de los hábitos más representativos dentro de los estilos de vida saludables. Desde diversos sectores especializados se destaca el papel que un ejercicio físico adecuado posee en la prevención de la mayoría de las alteraciones producidas por nuestra sociedad corroborando los efectos fisiológicos beneficiosos de una práctica de actividad física correcta, regular y sistematizada (Cruz, Fernández y González, 2007; García, Carrillo, Fernández y Sánchez, 2006; García, Cuevas, Hurtado, Sánchez y Martínez, 2005; Usán, 2016).

Desde esta perspectiva, numerosas investigaciones señalan que aquellos adolescentes que realizan una práctica habitual de actividad físico-deportiva se inician más tarde en el consumo recreativo de sustancias de abuso y/o sus prevalencias de consumo resultan inferiores con respecto a aquellos que no la practican (De Vries et al., 2006; Rodríguez et al., 2004; García et al., 2006; Pastor, Balaguer y García-Merita, 2006; Warren et al., 2008; Valdés y Yanci, 2016).

Sin embargo, otros estudios encuentran relaciones entre la práctica de la actividad física y consumo de alcohol y tabaco (Lorente, Souville, Griffet y Grélot, 2004; Menéndez y Fernández, 2015; Ruiz-Juan, Ruiz-Risueño y Zamarripa, 2012; Ruiz-Juan, Isorna, Ruiz-Risueño y Vaquero-Cristóbal, 2014; Vasters y Pillon, 2011).

Dos aspectos que resultan importantes en cualquier práctica físicodeportiva son la motivación y el autoconcepto de sus participantes, en cuanto pueden determinar su adherencia y/o evitación del abandono deportivo(García Calvo, 2006; Macarro, Romero y Torres, 2008; Mañas, Salvador, Boada y Agulló, 2007).

La motivación hacia la práctica deportiva es una de las variables psicológicas más estudiadas con una gran incidencia para la explicación de numerosos comportamientos en deportistas. La teoría de la autodeterminación (TAD) (Deci y Ryan, 2002) es una macro teoría relacionada con el desarrollo y funcionamiento de la personalidad en contextos sociales analizando el grado en que las conductas humanas son volitivas, propias de la elección personal, o autodeterminadas, asumiendo que las personas son organismos activos, con tendencias hacia el crecimiento y desarrollo psicológico que se esfuerzan por integrar sus experiencias en un sentido coherente.

De esta manera, la TAD actúa como un continuo que abarca diferentes grados de autodeterminación en la conducta, desde la no autodeterminada hasta la más autodeterminada (Moreno y Martínez, 2006). Durante todo ese recorrido, se abarcan tres tipos fundamentales de motivación (intrínseca, extrínseca y amotivación) con su propia estructura y reguladas por el sujeto de manera interna o externa.

La motivación intrínseca, hace referencia a los motivos propios del desarrollo de la actividad suponiendo el compromiso y disfrute del sujeto siendo un fin en sí misma (Deci y Ryan, 2000). La motivación extrínseca se basa en los motivos de práctica ajenos a la propia actividad siendo una tarea externa a la tarea que se está realizando. La amotivación, hace referencia a la falta de motivación para desarrollar una tarea o actividad en un contexto determinado.

Por lo tanto, la motivación es un elemento clave para lograr la adherencia y el compromiso en el deporte (Iso-Ahola y St.Clair, 2008) el cual es entendido como una disposición psicológica que representa el deseo y la decisión de seguir participando en él(Scanlan, Russell, Beals y Scanlan, 2003).

Muchos son los estudios que relacionan en población deportiva adolescente

conceptos asociados a la motivación como el clima motivacional y su relación con el compromiso deportivo (Torregrosa, Sousa, Viladrich, Villamarín Cruz, 2008), motivación y adhesión a la actividad física y deporte (Almagro, Sáenz, González-Cutre y Moreno, 2011), perspectivas de meta en el contexto deportivo (Castillo, Balaguer y Duda, 2002), motivación del logro en educación física (González-Cutre, Sicilia Camacho y Moreno, 2006) e incluso relacionan la motivación con aspectos personales como, auto-confianza, ansiedad y el estado de ánimo de los adolescentes (Cecchini, González, Carmona y Contreras, 2004).

Por otro lado, el autoconcepto físico es uno de los principales dominios del autoconcepto entendido desde un modelo jerárquico y multidimensional (Shavelson, Hubner y Stanton, 1976) ocupando un espacio propio dentro del mismo, dado que el autoconcepto general se compone del académico y no académico y este último, a su vez, incluye tanto el autoconcepto social como el físico.

El modelo tetradimensional del autoconcepto físico (Fox y Corbin, 1989, Goñi, Ruiz de Azúa y Rodríguez, 2006) es el más ampliamente aceptado en nuestros días incluyendo las dimensiones de competencia percibida (realidad dinámica que evoluciona paralelamente a la maduración de los sujetos y las experiencias de éxito o fracaso en los entornos de logro), fuerza física (percepción de la capacidad de generar tensión muscular ante una resistencia), atractivo físico (valoración de aspectos físicos) y autoconfianza (estimación del estado de la capacidad de rendimiento psico-físico). Así, este modelo ha sido ampliamente utilizado en psicología para explicar el autoconcepto físico en contextos deportivos.

De esta manera, el autoconcepto físico se relaciona con innumerables variables psicosociales tales como la actividad física en adolescentes(Hellin, 2007; Holgado, Soriano y Navas, 2009), hábitos alimentarios (Goñi y Rodríguez, 2004; Motl, McAuley, Birnbaum y Lytle, 2006) o bienestar psicológico (Merino y González, 2006).

Pese a todo ello, y siguiendo a Moreno, González-Cutre y Cervelló (2008) resultan necesarios más estudios que indaguen en el estudio de las relaciones entre la práctica de la actividad físico-deportiva y el consumo de drogas. De ahí que el principal objetivo de este estudio sea analizar la relación entre la motivación deportiva, el autoconcepto físico y el consumo de alcohol, tabaco y cannabis en futbolistas adolescentes.

De esta manera, y en base al objetivo anteriormente enunciado, se postulan dos hipótesis: (a) Los futbolistas que posean una mayor motivación intrínseca y mayores niveles de autoconcepto físico hacia la práctica del fútbol ofrecerán menores prevalencias de consumo de alcohol, tabaco y cannabis en una línea de conductas más adaptativas que, (b) Aquellos futbolistas que posean una mayor motivación extrínseca y amotivación unido a menores niveles de autoconcepto físico, relacionados con mayores prevalencias de consumo de alcohol, tabaco y cannabis en una línea de conductas menos adaptativas.

\section{Método}

\section{Diseño}

El presente trabajo, de acuerdo con Montero y León (2007), consistió en un estudio ex post-facto de carácter prospectivo con un diseño descriptivo simple, respondiendo a un muestreo aleatorio simple.

\section{Muestra}

El estudio estuvo compuesto por 512 adolescentes deportistas de género masculinos pertenecientes a 12 clubes de fútbol de la provincia de Zaragoza con edades comprendidas entre los 14 y 19 años $(M=16,01$; $\mathrm{DT}=1,465$ ) todos ellos federados y activos en la práctica deportiva.

\section{Instrumentos de evaluación}

Para recoger la información y aproximarnos al objetivo establecido se utilizaron tres cuestionarios.

Con la finalidad de percibir los motivos de práctica de los futbolistas se utilizó una versión traducida y adaptada al contexto específico del fútbol de la Sport Motivation Scale (Pelletier et al., 1995) validada y traducida al español por Núñez, Martín-Albo, Navarro y González (2006). Dicha escala se compone de 28 ítems repartidos en tres grandes motivaciones con sus respectivas subvariables de igual número deítems (4): motivación extrínseca, identificada $(\alpha=, 72)$, introyectada $(\alpha=, 78)$ y externa $(\alpha=, 79)$; motivación intrínseca, orientada hacia la práctica $(\alpha=, 82)$, aprendizaje $(\alpha=, 76)$ y perfeccionamiento $(\alpha=, 78)$ y, por último, amotivación $(\alpha=, 80)$ o, lo que es lo mismo, la falta de motiva- 
ción hacia la práctica deportiva. La fiabilidad del instrumento original denota un alfa de Cronbach de ,75 mientras que en nuestra investigación otorgó una prevalencia de ,76. El instrumento ha sido validado con diferentes muestras y culturas, mostrando una buena fiabilidad y validez en inglés (Pelletier et al., 1995; Pelletier y et al., 2001), francés (Brière y et al., 1995), portugués (Barreiro, 2005) y castellano (Núñez etal., 2006).

Para averiguar el autoconcepto físico de los futbolistas adolescentes se partió de la versión original de Physical Self-Questionnaire (CPSQ) de Fox y Corbin (1999) utilizando una versión traducida al castellano de Moreno, Cervelló, Vera y Ruiz (2007) orientada hacia jóvenes. El instrumento está compuesto por 30 ítems los cuales se dividen en cuatro subvariables: Competencia percibida (10ítems) $(\alpha=, 69)$; Atractivo físico (7 ítems) ( $\alpha=, 76)$; Fuerza física (6 ítems) $(\alpha=, 75)$ y Autoconfianza (4 ítems) $(\alpha=, 80)$. La fiabilidad de dicho instrumento originario es de,72 siendo la consistencia interna para nuestro estudio de ,73.

Por último, con la finalidad de percibir el consumo por parte de los futbolistas adolescentes se utilizó una versión adaptada de la Encuesta Estatal Sobre Uso de Drogas en Enseñanzas Secundarias (Estudes, 2010), promovida por la delegación del gobierno para el Plan Nacional sobre Drogas (PNSD). Concretamente, se tomaron las categorías generales del consumo de alcohol $(\alpha=, 74)$, tabaco $(\alpha=, 77)$ y cannabis $(\alpha=, 72)$ con tres opciones de respuesta: consumo habitual, ocasional o ausencia del mismo, mostrando una consistencia interna de ,76.

\section{Procedimiento}

En la realización del estudio se contó con la aprobación de los clubes de fútbol y de los padres/tutores de los futbolistas por medio de consentimiento informado para poder participar en la investigación. Un día de la semana acordado previamente sellevó a cabo la cumplimentación de los cuestionarios en cada uno de los clubes. Una vez recogidos los datos, se procesaron y analizaron mediante el programa estadístico IBM SPSS v21.0. Todos los sujetos y sus padres/tutores fueron previamente informados de la naturaleza del estudio participando de manera voluntaria y respetando las directrices éticas de la Declaración de Helsinki (AMM, 2000) en todos sus términos.

\section{Análisis de datos}

Se procedió a realizar una estadística descriptiva con la finalidad de hallar datos sociodemográficos y la media aritmética en función de las diferentes variadas estudiadas. Posteriormente se llevaron a cabo correlaciones y análisis de varianza entre las variables motivación, autoconcepto físico y consumo. Por último, mediante el análisis de cluster, se clasificaron a los sujetos del estudio, según sus características específicas, en diferentes grupos cuyas puntuaciones fueron significativas entre sí. Para todas las operaciones, se tuvo en cuenta un nivel de significación pd»0,05 trabajándose con un nivel de confianza del 95\%.

\section{Resultados}

A continuación se presentan los resultados obtenidos en las diferentes variables estudiadas

\section{Variables descriptivas}

Tal y como se refleja en la tabla 2, las motivaciones intrínsecas (orientadas hacia la práctica, aprendizaje y perfeccionamiento) obtuvieron mayores puntuaciones que las extrínsecas, siendo la motivación introyectada $(\mathrm{M}=3,74)$ la mayor media entre ellas. La amotivación $(\mathrm{M}=1,74)$, resultó la menos puntuada por los deportistas adolescentes. Por parte del autoconcepto físico, la autoconfianza $(\mathrm{M}=3,96)$ y la competencia percibida $(\mathrm{M}=3,20)$ fueron las variables con las medias más altas.

Por su parte, atendiendo al consumo, se denotó el alcohol como la sustancia más consumida entre los futbolistas adolescentes tanto a nivel habitual ( $\mathrm{N}=152 ; 29,7 \%)$ como ocasional ( $\mathrm{N}=247 ; 48,2 \%$ ) seguida por el tabaco (véase tabla 3). El consumo de cannabis resultó ser menos acentuado que las dos anteriores con escasas prevalencias de consumo habitual ( $\mathrm{N}=34 ; 6,6 \%)$ así como una considerable ausencia del mismo $(\mathrm{N}=366 ; 71,5 \%)$.

\section{Relación entre variables deportivas y consumo}

Analizando las variables deportivas y de consumo se apreciaron correlaciones significativas entre varias de ellas (véase Tabla 4). De esta manera, destacó la significación de motivaciones intrínsecas (especialmente entre las orientadas hacia la práctica y el aprendizaje) con el consumo de alcohol, tabaco y cannabis, a la vez que lo hizo con la motivación extrínseca identificada $\left(-, 146^{* *}\right)$ y la falta de motivación $\left(, 143^{* *}\right)$. Por parte del autoconcepto físico, tan sólo la competencia percibida mostró relación significativa con el consumo de las tres sustancias estudiadas.

\section{Influencia de variables deportivas en consumo}

Realizando un análisis de varianza se observaron la influencia de las variables deportivas en consumos (véase tabla 5) donde cabe destacar la influencia que las motivaciones intrínsecas ejercen sobre el consumo de alcohol, tabaco y cannabis. A su vez, la motivación extrínseca identificaday amotivación se mostraron significativas con el consumo de alcohol, tabaco y cannabis, del mismo modo que la extrínseca introyectada con tabaco $\left(3,706^{* *}\right)$ y cannabis $\left(4,859^{* *}\right)$. Tal y como sucediera en el apartado anterior, la competencia percibida vuelve a influir en los consumos de las tres sustancias psicoactivas.

\section{Patrones de conducta entre variables deportivas y consumo}

Por último, se procedió a clasificar a los sujetos en grupos homogéneos en función de las variables deportivas y consumo realizando un análisis de cluster, el cual tiene por objetivo la clasificación de los individuos en grupos significativos. De esta manera, según se puede observar en la Tabla 6, se establecieron tres grupos significativos en función de sus medias.

A continuación, se muestra de manera específica las diferentes medias de cada variable en función a los tres grupos significativos anteriores (Tabla 7).

- $\quad 1^{\circ}$ Grupo ( $\left.N=130 ; 25,24 \%\right)$ Caracterizado por conductas menos adaptativas: Las motivaciones intrínsecas, competencia percibida y autoconfianza se dispusieron por debajo de la media en detrimento de un mayor consumo de alcohol, tabaco y cannabis así como prevalencias superiores de amotivación.

- $\quad 2^{\circ}$ Grupo (N=154; 30,13\%) Caracterizado por conductas mixtas: Las variables deportivas de motivación y autoconcepto se dispusieron en su mayoría por encima de la media, resultando prevalencias ligeramente superiores a ella en los consumos de alcohol, tabaco y cannabis.

- $\quad 3^{\circ} \mathrm{Grupo}(\mathrm{N}=229 ; 44,81 \%)$ Caracterizado por conductas más adaptativas: Las medias de este grupo en motivaciones intrínsecas, competencia percibida y autoconfianza fueron superiores a la media total resultando las extrínsecas y amotivación por debajo de ella. Todo ello unido a un considerable descenso de las prevalencias de alcohol, tabaco y cannabis por parte de los futbolistas adolescentes que componían este grupo.

\section{Discusión}

El objetivo del estudio fue analizar la relación entre motivación, autoconcepto físico y consumo de drogas en futbolistas adolescentes.

La primera hipótesis que se postuló fue, precisamente, que aquellos futbolistas adolescentes con una mayor motivación intrínseca y mayores niveles de autoconcepto físico hacia la práctica del fútbol ofrecerían menores niveles de consumo de alcohol, tabaco y cannabis en una línea de conductas más adaptativas. Dicha hipótesis se cumplió; los resultados de nuestra investigación revelaron la relación entre motivaciones predominantemente intrínsecas (hacia la práctica, aprendizaje y perfeccionamiento) con una mayor competencia percibida y autoconfianza en el autoconcepto físico unido ligado a menores 
Tabla 1.

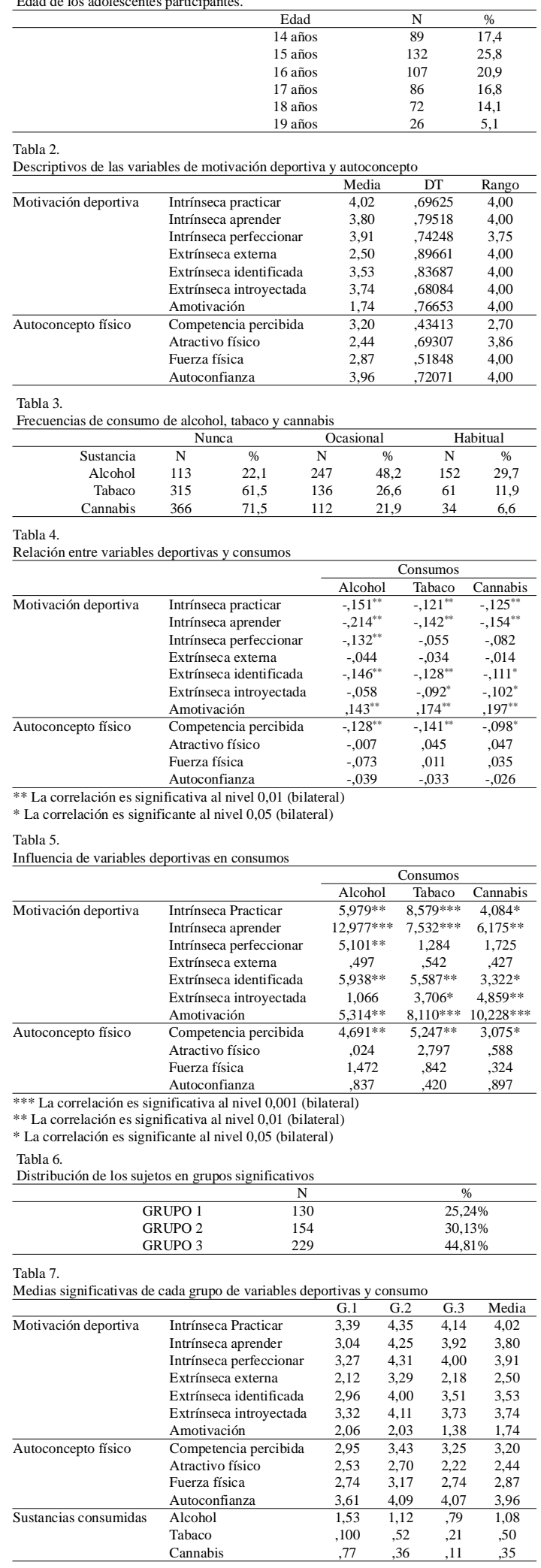

prevalencias de consumo de alcohol, tabaco y cannabis. Por un lado, diversos estudios ponen de manifiesto la relación entre motivación y consumo de sustancias. Moreno, González-Cutre y Cervelló (2008) determinan que aquellos deportistas con motivaciones más autodeterminadas e intrínsecas predecían positiva y significativamente los motivos de salud y ausencia de consumos. De esta manera, los sujetos con ausencia o escasa prevalencia de consumo se encontraban más intrínsecamente motivados hacia la práctica físico-deportiva. Otros estudios asocian un menor consumo de tabaco y alcohol con una alta motivación intrínseca y mayor práctica físico-deportiva en edad extraescolar (Inglés et al., 2007; Llamas, 2009; Usán 2017). En una misma línea, Leyton, Jiménez, Domínguez y Corzo (2012) manifiestan la relación de una ausencia de consumo de tabaco en relación a motivaciones intrínsecas y autorreferenciales unidas a hábitos de salud favorables como un adecuado descanso y alimentación. Por otro lado, otras investigaciones ponen de relieve la relación de la motivación y el autoconcepto físico en la práctica deportiva. En esta línea, Duda y Ntoumanis (2005) mostraron que la práctica deportiva extraescolar de los jóvenes, cuando se realiza buscando criterios más adaptativos, dejando de lado inadecuados hábitos de salud como el consumo de sustancias, se relaciona con un conjunto de consecuencias psicológicas positivas que incluyen mejoras del autoconcepto físico traducido en una mayor consideración de la competencia percibida, autoconfianza y persistencia en la práctica de actividades físicas, entre otras. A su vez, Lázaro (2011) argumenta que los niveles de condición física relacionados con la salud de los adolescentes suponen uno de los factores que mayor importancia poseen como elemento disuasorio del consumo de alcohol. Por su parte, Moreno, Moreno y Cervelló (2009) muestran relaciones positivas entre motivaciones intrínsecas, condición física y competencia percibida ligado a la ausencia de consumo tabaco concluyendo que, la práctica físico-deportiva, predice la autoestima en particular y el autoconcepto físico en general, revelándose como un factor muy importante para la salud, tanto física como psicológica (Moreno, Cervelló y Moreno, 2008; Portolés y González, 2016; Rees y Sabia, 2010).

La segunda hipótesis de nuestro estudio aludía a la posible relación entre motivaciones extrínsecas, amotivación y mayores niveles de consumo de alcohol, tabaco y cannabis en una línea de conductas menos adaptativas. Así, desde una vertiente menos autodeterminada, los resultados de nuestra investigación corroboran la segunda hipótesis dilucidando relaciones significativas entre estas variables. De este modo, diferentes trabajos aluden a una mayor medida de los motivos extrínsecos en la práctica físico-deportiva en coincidencia con el consumo de sustancias ligado a perfiles de conducta más desadaptativos que, incluso, pueden derivar en el abandono deportivo (Ruiz-Risueño, 2011; Vasters y Pillon, 2011). Los trabajos de Moreno, González-Cutre y Cervelló (2008) determinan que aquellos deportistas que adquieren una motivación menos autodeterminada en la práctica deportiva predicen significativamente hábitos de salud poco consistentes y consumo de sustancias durante la adolescencia. De igual manera que los futbolistas adolescentes de nuestro estudio, los deportistas que consumen habitualmente alcohol y tabaco reflejan motivaciones de predominio extrínseco. Moreno y Cervelló (2009) muestran relaciones positivas entre motivaciones puramente extrínsecas, condición física y consumo de alcohol al igual que la concordancia de la imagen personal y atractivo físico del deportista en comportamientos más desadaptativos. A su vez, el consumo de cannabis se ha relacionado peyorativamente con la motivación y adhesión hacia la práctica deportiva. De estemodo, Villalbí, Suelves, Saltó y Cabezas (2011), manifiestan la implicación y consecuencias del consumo de cannabis en el rendimiento deportivo de los futbolistas (mayor fatigabilidad, alteración de recuperación, dificultad de adaptación cardíaca, sensación de deshidratación...) mermando su motivación intrínseca hacia la práctica deportiva. A su vez, la relación entre el autoconcepto, como constructo multidimensional y el consumo de sustancias es un tema ampliamente estudiado, sobre todo en el periodo de la adolescencia (Calafat et al., 2011; Echeburúa y Corral, 2010; Prado y Pantin, 2011) en el que, tradicionalmente, se ha considerado el bajo autoconcepto como un clásico factor de riesgo para el consumo de sustancias (Lamb y Crano, 2013; Pérez, López, Cuesta y Caballero, 2005). Algunos autores concluyen que un bajo autoconcepto físico, social o emocional está relacionado con altos niveles de consumo (Kavas, 2009; Zamboaga, Schwartz, Jarvis y Van Tyne, 2009) tal y como se desprende de nuestros resultados; otros no encuentran relación (Kokkevi, Richardson, Florescu, Kuzman y Stergar, 2007). Sin embargo, resultan escasos los estudios que relacionen de manera directa el autoconcepto físico en deportistas con el consumo de sustancias a pesar de la importancia del mismo como estimador fiable del bienestar 
de la persona, sobre todo en la adolescencia y juventud (Garaigordobil y Durá, 2006; Pastor, Balaguer y García 2006) denotando la relación entre el autoconcepto físico y un estilo de vida saludable (Morsunbul, 2013; Tarbell y Li, 2013) alejado del consumo de drogas recreativas de manera habitual y/o prolongada tal y como se denota en nuestro estudio.

Por todo esto, aspectos referidos a motivación y autoconcepto físico, tal y como venimos comentando, influyen y desempeñan una labor relevante sobre las conductas de consumo (Jiménez, Cervelló, García-Calvo, Santos-Rosa e Iglesias, 2007; Rubak, Sandbaek, Lauritzen y Christensen, 2005) resultando fundamental atender a ellos para una mayor eficiencia en la prevención e intervención de las mismas en aras de una mayor adherencia y disfrute de la actividad físico-deportiva (Agulló-Calatayud, 2008; Bartík, 2012; Hodge, Hargreaves, Gerrard y Lonsdale, 2013; Pérez-Milena, Olmedilla, Mesa, Jiménez y Martínez, 2010; Prieto, 2015; Roessler, 2009).

\section{Limitaciones del estudio}

Las limitaciones del estudio pueden residir en el diseño transversal del mismo tomando los datos en un momento espacio temporal dado. A su vez, los clubes encuestados responden de una manera aleatoria a su inclusión en el estudio no tomando una muestra uniforme de todos los barrios o zonas de la ciudad afectando, del mismo modo, a las posibles diferencias entre sus niveles deportivos y otras cuestiones sociales. Ala par, las prevalencias de consumo, motivación o autoconcepto físico pueden variar de un año a otro, e incluso dentro de un mismo año, en cuanto los adolescentes avanzan en la etapa de la adolescencia.

\section{Prospectivas de futuro}

Como prospectivas de futuro resultarían interesantes modelos longitudinales que evalúen y contrasten la creación o aplicación de programas de intervención para ver la influencia de los mismos en el tiempo. Del mismo modo, sería interesante incluir diferencias por género en el creciente fútbol femenino para comprobar si existen diferencias significativas con el fútbol masculino. Por último, una muestra de mayor abanico de edades extendiéndonos al futbol regional podría otorgar una visión más holística del fútbol en categorías no adolescentes.

\section{Implicaciones prácticas}

Este trabajo, puede tener unas implicaciones de carácter práctico que desemboquen en unas estrategias didácticas a utilizar sobre deportistas adolescentes, a través de entrenadores o monitores deportivos orientadas hacia conductas autodeterminadas como la prevención de consumo de drogas y la promoción de motivaciones intrínsecas, competencia percibida, autoconfianza, etc., que conduzcan a una adecuada práctica de la actividad físico-deportiva. A su vez, programas de intervención dirigidos por profesionales del ámbito en la apuesta de la Administración y/o los propios clubes deportivos por el trabajo con sus adolescentes en las variables acaecidas puede ayudar a contribuir a la formación integral y educacional del deportista no reduciéndola sólo a la deportiva.

\section{Referencias}

Agulló-Calatayud, V., González-Alcaide, G, Valderrama-Zurián, J.C. y AleixandreBenavent, R. (2008). Consumption of anabolic steroids in sport, physical activity and as a drug of abuse: An analysis of the scientific literature and areas of research. British Journal of Sports Medicine, 42(2), 103-109.

Almagro, B.J., Sáenz, P., González-Cutre, D. y Moreno, J.A. (2011). Clima motivacional percibido, necesidades psicológicas y motivación intrínseca como predictores del compromiso deportivo en adolescentes. Revista Internacional de Ciencias del Deporte, 25(7), 250-265.

Becoña, E. (2007). Bases psicológicas de la prevención del consumo de drogas. Papeles del Psicólogo, 28(1), 11-20.

Becoña, E. (2014). Heroína, cocaína y otras drogas de síntesis. Madrid: Síntesis.

Becoña, E., Fernández, E., Calafat, A. y Fernández-Hermida, J.R. (2014). Apego y consumo de sustancias en la adolescencia: Una revisión de aspectos conceptuales y metodológicos. Adicciones, 26, 77-86.

Boyle, A.M. y O'Sullivan, L. F. (2013). The influence of dating anxiety on normative experiences of dating, sexual interactions, and alcohol consumption among canadians middle adolescents. Journal of Youth Studies, 16(2), 222-236.

Bravo, R., Echeburúa, E. y Aizpiri, J. (2008). Diferencias de sexo en la dependencia del alcohol: dimensiones de personalidad, características psicopatológicas y trastornos de personalidad. Psicothema, 20, 218-223.

Calafat, A., Mantecón, A., Juan, M., Adrover, D., Blay, N., Hughes, K. y Rosal, F. (2011). Violent behavior, drunkenness, drug use and social capital in nightlife.Psychosocial Intervention, 20, 45-51.

Capdevila, A., Bellmunt, H. y Hernando, C. (2015). Estilo de vida y rendimiento académico en deportistas. Comparación entre deportistas y no deportistas. Retos. Nuevas Tendencias en Educación Física, Recreación y Deporte, 27(1), 28-33.

Cecchini, J.A., Gónzalez, C., Carmona A.M. y Contreras O. (2004). Relaciones entre clima motivacional, la orientación de meta, la motivación intrínseca, la auto-confianza, la ansiedad y el estado de ánimo en jóvenes deportistas. Psicothema, 16(1), 104-109.

Cruz, P.J., Fernández, E. y González, G.M. (2007). El deporte como método preventivo en el uso de las drogas: teoría y práctica. Revista Digital Wanceulen, 3, http:// www.wanceulen.com/revista/PDF/n3/deporte_preventivo_drogas.pdf, extraído el 2/X/2016.

Danjoy, D., Ferreira, P.S. y Pillon, S.C. (2010). Conocimientos y prácticas sobre el consumo de tabaco en estudiantes de pregrado de farmacia, Lima, Perú. Latinoamericana Enfermagem, 18.

De Vries, H., Dijk, F.,Wetzels, J., Mudde, A., Kremers, S. y Ariza C. (2006). The European Smoking prevention Framework Approach (ESFA), effects after 24 and 30 months. Health Education Research, 21(1), 116-132.

Deci, E.L. y Ryan, R.M. (2000).The "what” and "why” of goal pursuits: Human needs and the self-determination of behavior. Psychological Inquiry, 11, 227-268.

Deci, E.L. y Ryan, R.M. (2002).Self-determination research: Reflections and future directions. In E.L. Deci y R.M. Ryan (Eds.), Handbook of self-determination research. (pp. 431-441). University of Rochester Press.

Drobnie, F. (2003). El consumo del cannabis y el deporte. Archivos de Medicina del Deporte, 97(2), 385-386.

Duda, J.L. (2005). Motivation in Sport: The Relevance of Competence and Achievement Goals. En A. J. Elliot y C. S. Dweck (Eds.), Handbook of competence and motivation (pp. 273-308).New York: Guilford Publications.

Dunn, M. y Thomas, J.O. (2012). A risk profile of elite australian athletes who use illicit drugs. Addictive Behaviors, 37(1), 144-147.

Echeburúa, E. y Corral, P. (2010).Adicción a las nuevas tecnologías y a las redes sociales en jóvenes: Un nuevo reto. Adicciones, 22, 91-96.

Encuesta Estatal sobre Uso de Drogas en Enseñanzas Secundarias (ESTUDES) (2014). Madrid: Ministerio del Interior. Plan Nacional sobre Drogas.

Fox, K.R. y Corbin, C.B. (1989). The physical self-perception profile: Development and preliminary validation. Journal of Sport and Exercise Psychology, 11, 408-430.

Garaigordobil, M. y Durá, A. (2006). Psychopathological symptoms, behavioural problems, and self-concept/self-esteem: A study of adolescents aged 14 to 17 years old. Annuary of Clinical and Health Psychology, 1, 53-63.

García, P., Carrillo, A., Fernández, A. y Sánchez, J.M. (2006). Factores de riesgo en la experimentación y el consumo de tabaco en estudiantes de 12 a 14 años. Actitudes ante el tabaco en los grupos de presión. Revista de Atención Primaria, 37, 392-399.

García, P., Cuevas, M. D., Hurtado, S., Sánchez, J. y Martínez, R. (2005). Comparación de las actitudes, percepción, entorno y experimentación con el tabaco en cuatro poblaciones de $1^{\circ} \mathrm{de}$ ESO de la Región de Murcia. ICongreso Internacional de Enfermería Comunitaria, Madrid.

García-Calvo, T. (2006). Motivación y comportamientos adaptativos en jóvenes futbolistas. Tesis Doctoral. Universidad de Extremadura.

Gilman, S.E., Rende, R., Boergers, J., Abrams, D.B., Buka, S.L., Clark, M.A., Niaura, R.S. (2009). Parental smoking and adolescent smoking initiation: an intergenerational perspective on tobacco control. Pediatrics, 123, 274-281.

Gómez-Fraguela, J.A., Fernández, N., Romero, E. y Luengo, A. (2008). El botellón y el consumo de alcohol y otras drogas en la juventud. Trastornos Adictivos, 20(2), 211-217.

Goncy, E.A. y Mrug, S. (2013). Where and when adolescents use tobacco, alcohol, and marijuana: Comparisons by age, gender, and race. Journal of Studies on Alcohol and Drugs, 74(2), 288-300.

González-Cutre, D., Sicilia, A. y Moreno, J. A. (2006). Predicción de las razones de responsabilidad para ser disciplinado en el deporte a través de las orientaciones de meta y los climas motivacionales. En M. A. González, J. A. Sánchez y A. Areces (Eds.), IV Congreso de la Asociación Española de Ciencias del Deporte (pp. 736739). A Coruña: Xunta de Galicia.

Goñi, A. y Rodríguez, A. (2004). Trastornos de conducta alimentaria, práctica deportiva y autoconcepto físico en adolescentes. Actas Españolas de Psiquiatría, 32(1), 2936.

Goñi, A., Ruiz de Azúa, S., y Rodríguez, A. (2006). Cuestionario de Autoconcepto Físico. Manual. Madrid: EOS.

Hellín, M.G. (2007). Motivación, autoconcepto físico, disciplina y orientación disposicional en estudiantes de educación física. Tesis Doctoral. Universidad de Murcia.

Hock, H. y Weil, D.N. (2012). On the dynamics of the age structure, dependency, and consumption. Journal of Population Economics, 25(3), 1019-1043.

Hodge, K., Hargreaves, E.A., Gerrard, D. y Lonsdale, C.(2013). Psychological mechanisms underlying doping attitudes in sport: Motivation and moral disengagement. Journal of Sport and Exercise Psychology, 35(4), 419-432. 
Holgado, F.P., Soriano, J.A. y Navas L. (2009). Cuestionario de autoconcepto físico (CAF): Análisis factorial confirmatorio y predictivo sobre el rendimiento académico global y específico del área de educación física. Acción psicológica, 6(2), 93102.

Inglés, Martínez-González, García-Fernández, Torregrosa y Ruiz-Esteban (2012). Prosocial behavior and self-concept of spanish students of compulsory secondary education. Revista de psicodidácica, 17, 135-156.

Inglés, C.J., Delgado, B., Bautista, R., Torregrosa, M.S., Espada, J.P., García-Fernández, J.M., Hidalgo, M.D. y García-López, L.J. (2007). Factores psicosociales relacionados con el consumo de alcohol y tabaco en adolescentes españoles. Internationa Journal of Clinical and Health Psychology, 7, 403-420.

Iso-Ahola, S.E. y St.Clair, B. (2008). Toward a theory of exercise motivation. Quest, 52, 131-147.

Jiménez, R., Cervelló, E., García-Calvo, T., Santos-Rosa, F.J., Iglesias, D. (2007). Estudio de las relaciones entre motivación, práctica deportiva extraescolar y hábitos alimenticios y de descanso en estudiantes de Educación Física. International Journal of Clinical and Health Psychology, 7(2), 385-401.

Kavas, A.B. (2009). Self-esteem and health-risk behaviours among Turkish late adolescents. Adolescence, 44(173), 187-198.

Kokkevi, A., Richardson, C., Florescu, S., Kuzman, M. y Stergar, E. (2007). Psychosocia correlates of substance use in adolescence: A cross-national study in six European countries. Drug and Alcohol Dependence, 86, 67-74.

Kyle, T., Mendo, A., Garrido, R. y Sánchez, V.M. (2016). Efectos de la actividad física en el autoconcepto y la autoeficacia en preadolescentes. Retos. Nuevas tendencias en Educación Física, Deporte y Recreación, 29(1), 61-65.

Hernández-Soriano, O., Font-Mayolas, S. y Gras, M.E. (2015). Policonsumo de drogas y su relación con el contexto familiar y social en jóvenes universitarios. Adicciones, 27(3), 205-213

Lamb, C.S. y Crano, W.D. (2013). Parents'beliefs and children's marijuana use: Evidence for a self-fulfilling prophecy effect. Addictive Behaviors, 39, 127-132.

Lázaro, C.J. (2011). Hábitos de consumo de alcohol y su relación con la condición física saludable en adolescentes de la región de Murcia. Tesis Doctoral. Universidad de Murcia.

Leyton, M., Jiménez, R., Domínguez, F.J. y Corzo., H. (2012). Análisis correlacional entre la teoría de la autodeterminación y variables de estilos de vida saludables: descanso, tabaco y alimentación. IV Congreso internacional de ciencias del deporte y la Educación Física. (VIII Seminario nacional de nutrición, medicina y rendimiento deportivo). Pontevedra.

Llamas, L.S. (2009). Motivación autodeterminada y clima motivacional en educación física. Tesis Doctoral. Universidad de Elche.

Macarro, J., Romero, C. y Torres, J.(2008). Motivos de abandono de la práctica de actividad físico-deportiva en los estudiantes de Bachillerato de la provincia de Granada. Revista de Educación, 353, 495-519.

Mañas, M.A., Salvador, C., Boada, J. y Agulló, A. (2007). La satisfacción y el bienestar psicológico como antecedentes del compromiso organizacional. Psicothema, 19 395-400.

Méndez, A., Martínez, D. y Valverde, J.J. (2016). Valoración del alumnado y profesorado del material convencional y auto-construido: estudio longitudinal de diseño cruzado en Educación Deportiva. Retos. Nuevas tendencias en Educación Física, Deporte y Recreación, 30(2), 16-30.

Menéndez, J.I. y Fernández, J. (2015). Hábitos de alimentación y actividad física de Menores Extranjeros No acompañados (MENAs): un estudio de caso. Retos. Nuevas tendencias en Educación Física, Deporte y Recreación, 28(2), 33-39.

Merino, B. y González, E. (2006). Actividad física y salud en la infancia y adolescencia. Guía para todas las personas que participan en su educación. Madrid: Ministerio de Educación y Ciencia y Ministerio de Sanidad y Consumo.

Moreno, J. A y Martínez, A. (2006). Importancia de la Teoría de la Autodeterminación en la práctica físico-deportiva: Fundamentos e implicaciones prácticas. Cuadernos de Psicología del Deporte, 6(2), 39-54.

Moreno, J.A., Cervelló, E. y Moreno, R. (2008). Importancia de la práctica físico-deportiva del género en el autoconcepto físico de los 9 a los 23 años. International Journal of Clinical and Health Psychology, 8(1), 171-183.

Moreno, J.A., González-Cutre, D. y Cervelló, E.M. (2008). Motivación y salud en la práctica físico-deportiva: diferencias según el consumo de alcohol y tabaco. International Journal of Clinical and Health Psychology, 8, 483-494.

Moreno, J.A., Moreno, R. y Cervelló, E. (2009). Relación del autoconcepto físico con las conductas de consumo de alcohol y tabaco en adolescentes. Adicciones, 21, 147154.

Morsunbul, U. (2013). An investigation of the relationships between agency, identity formation and life satisfaction in adolescence period. Dusunen Adam, 26(2), 164 170.

Motl, R., McAuley, E., Birnbaum, A. y Lytle, L. (2006). Naturalle occurring changes in time spent watching televisión are inversely related to frequency of physical activity during early adolescence. Journal of Adolescence, 29, 19-32.

Ntoumanis, N. (2005). A prospective study of participation in optional school physical education using a self-determination theory framework. Journal of Educationa Psychology, 97(3), 444-453.

Observatorio Español de la Droga y las Toxicomanías (OEDT) (2016). Informe 2016 : Alcohol, tabaco y otras drogas ilegales en España. Madrid: Ministerio del Interior.

Oliva, A., Parra A. y Sánchez-Queija, I. (2006). Consumo de sustancias durante la adolescencia: Trayectorias evolutivas y consecuencias para el ajuste psicológico. International Journal of Clinical and Health Psychology, 8(1), 153-169.
Pastor, Y., Balaguer, I. y García-Merita, M. (2006). Relaciones entre el autoconcepto y el estilo de vida saludable en la adolescencia media: un modelo exploratorio. Psicothema, 18 (1), 18-24.

Pérez, E.J., López, M.P. Cuesta, S.E. y Caballero, C.G. (2005). Validación del cuestionario de variables de interacción psicosocial (VIP): Hacia un modelo de tratamiento de las conductas adicitivas guiado por la personalidad. Trastornos adictivos, 7, 166186

Pérez-Milena, A., Olmedilla, M.R., Mesa, I., Jiménez, I., Martínez, M.L., y Pérez-Milena, R. (2010). Motivaciones para el consumo de alcohol entre adolescentes de un instituto urbano. Atencion Primaria, 42(12), 604-611.

Plan Nacional Sobre Drogas (PNSD) (2016). Alcohol, tabaco y otras drogas ilegales en España. Madrid: Ministerio del Interior.

Portolés, A. y González, J. (2016). Actividad física y niveles de burnout en alumnos de la E.S.O. Retos. Nuevas tendencias en Educación Física, Deporte y Recreación, 29(1), 95-99.

Prado, G. y Pantin, H. (2011). Reducing substance use and HIV health disparities among hispanic youth in the USA: Familias Unidas program of research. Psychosocial Intervention, 20, 63-73.

Prieto, J.M. (2015). Variables deportivas y personales en la ocurrencia de lesiones deportivas. Diferencias entre deportes individuales y colectivos. Retos. Nuevas tendencias en Educación Física, Deporte y Recreación, 28(2), 21-25.

Rees, D.I. y Sabia, J.J. (2010). Sports participation and academic performance: Evidence from the national longitudinal study of adolescent health. Economics of Education Review, 29(5), 751-759.

Roessler, K. (2009). «Sport makes me high» The role of physical activity in treatment for drug abuse. Zeitschrift Fur Sportpsychologie, 16(4), 151-155.

Rubak, S., Sandbaek, A., Lauritzen, T. y Christensen, B. (2005). Motivational interviewing: A systematic review and meta-analysis. British Journal of General Practice, 55, 305-312.

Ruiz-Juan, J.M., Isorna, M., Ruiz-Risueño, J.R. y Vaquero, R. (2014). Consumo e ingesta de alcohol en españoles mayores de 16 años y su relación con la actividad físicodeportiva, la familia y el consumo de tabaco. Revista Iberoamericana de Psicología del Ejercicio y el Deporte, 9(2), 339-372.

Ruiz-Risueño, J. (2011). Actividad físico-deportiva y contexto familiar: variables predictoras de consumo de alcohol y tabaco. Estudio comparativo entre adolescentes españoles y mexicanos de enseñanza secundaria. Tesis Doctoral. Universidad de Murcia.

Ruiz-Risueño, J., Ruiz-Juan, F, Zamarripa, J.I. (2012). Alcohol y tabaco en adolescentes españoles y mexicanos y su relación con la actividad físico-deportiva y la familia. Panam Salud Publica, 31(3), 211-220.

Ruiz-Risueño, J. y Ruiz-Juan, F. (2015). Actividad físico-deportiva y contexto familiar: variables predictoras de consumo de tabaco entre adolescentes españoles. Revista Iberoamericana de psicología del ejercicio físico y del deporte, 10(1), 121-131.

Salomó A., Gras M.E. y Font-Mayolas S. (2010). Patrones de consumo de alcohol en la adolescencia. Psicothema, 22(2), 189-195.

Scanlan, T.K., Russell, D.G., Beals, K.P. y Scanlan, L.A. (2008). Project on Elite Athlete Commitment (PEAK): Introduction and Methodology. Journal of Sport and Exercise Psychology, 25, 360-376.

Shavelson, R.J., Hubner, J.J., y Stanton, J.C. (1976). Self-concept: Validation of construct interpretations. Review of Elementary School Children, 46, 407-441.

Tarbell, S.E. y Li, B.U.K. (2013). Health-related quality of life in children and adolescents with cyclic vomiting syndrome: A comparison with published data on youth with irritable bowel syndrome and organic gastrointestinal disorders. Journal of Pediatrics, 163(2), 493-497.

Torregrosa, M., Sousa, C., Viladrich., C., Villamartín., F y Cruz., J., (2008). El clima motivacional y el estilo de comunicación del entrenador como predictores del compromiso en futbolistas jóvenes. Psicothema, 20(2), 254-259.

Usán, P. (2014). Consumo de drogas y variables psicológicas en futbolistas adolescentes. Tesis doctoral. Universidad de Zaragoza, España.

Usán, P., Salavera, C., Murillo, V. y Mejías, J.J.(2016). Relación entre motivación, compromiso y autoconcepto en adolescentes: un estudio con futbolistas. Cuadernos de Psicología del Deporte, 16(1), 199-210.

Usán, P., Salavera, C., Murillo, V.y Álvarez, J. (2017). Creencias y percepciones de éxito en futbolistas adolescentes. Retos. Nuevas Tendencias en Educación Física, Deporte y Recreación, 31(1), 207-211.

Valdés, P. y Yanci, J. (2016). Análisis de la condición física, tipo de actividad física realizada y rendimiento académico en estudiantes de educación secundaria. Retos. Nuevas Tendencias en Educación Física, Deporte y Recreación, 30(2), 64-69.

Vasters, G.P. y Pillon, S.C. (2011). Drugs use by adolescents and their perceptions about specialized treatment adherence and dropout. Revista Latino Americana De Enfermagem, 19(2), 317-324.

Vasters, G.P. y Pillon, S.C. (2011). Drugs use by adolescents and their perceptions about specialized treatment adherence and dropout. Revista Latino Americana De Enfermagem, 19(2), 317-324.

Villalbí, J., Suelves, J., Saltó., E. y Cabezas, C. (2011). Valoración de las encuestas a adolescentes sobre consumo de tabaco, alcohol y cannabis en España, Adicciones, 23(1), 11-16.

Warren, C.W., Jones, N.R., Peruga, A., Chauvin, J., Baptiste, J.P. y Costa de Silva,V. (2008) Global Routh tobacco surveillance, 2000-2007. MMWR SurveillSumm, 57(1), 128.

Zamboaga, B.L. Schwartz, S.J., Jarvis, L.H. y Van Tyne, K. (2009). Acculturation and substance use among Hispanic early adolescents: Investigating the mediating roles of acculturative stress and self-esteem. The Journal of Primary Prevention, 30 315-333. 